\title{
Unknown ESUR cases 2004
}

\author{
R. H. Cohan, ${ }^{1}$ N. C. Cowan, ${ }^{2}$ J. H. Ellis ${ }^{1}$ \\ ${ }^{1}$ Department of Radiology, University of Michigan Hospital, Room B1D502G, 1500 East Medical Center Drive, Ann Arbor, \\ Michigan 48109-0030, USA \\ ${ }^{2}$ The Churchill Hospital, Oxford University, Oxford, 0X3 7LJ England, United Kingdom
}

\section{Abstract}

The authors present 14 cases from the film interpretation session of the 11th annual meeting of the European Society of Urogenital Radiology presented in September, 2004. The cases demonstrate the imaging findings, differential diagnoses, and clinical relevance of a wide variety of genitourinary tract diseases. The cases include examples of benign and malignant urinary tract neoplasms, inflammatory processes, vascular diseases, traumatic injuries, and congenital anomalies.

Key words: Genitourinary tract neoplasms-Genitourinary tract inflammation-Genitourinary tract congenital anomalies - Genitourinary tract traumaGenitourinary tract vascular diseases

The case reports that follow represent a compilation of those cases that were presented as part of the Film Reading Session at the 11th Annual Symposium on Urogenital Radiology held by the European Society of Urogenital Radiology in Santiago de Compostela, Spain on 10 September 2004.

\section{Case 1: a 47-year-old male with hematuria (Fig. 1)}

\section{Description of findings}

An unenhanced computed tomographic (CT) image through the midabdomen demonstrates a large heterogeneous left renal mass. The mass contains several small areas of extremely low attenuation, one of which measures $-44 \mathrm{HU}$, indicating the presence of fat. In addition, there are multiple punctate high-attenuation foci consistent with calcifications.

Film Reading Session from the 11th Annual Symposium of the European Society of Urogenital Radiology, Santiago de Compostrela, Spain, 9-12 September 2004

Correspondence to: R. H. Cohan; email: rcohan@umich.edu

\section{Discussion}

In the vast majority of cases, the presence of macroscopic fat within a renal mass indicates that the mass represents an angiomyolipoma. Only a few pixels of fat are required to make this diagnosis, even if the major portion or almost all of the mass contains soft tissue attenuation components. However, calcifications are exceedingly rare in angiomyolipomas. If calcification is detected on CT in a fat-containing renal mass, the mass is more likely to represent an unusual renal cancer, with the fat and calcification arising from osseous metaplasia [1, 2]. There are several other extremely rare instances in which macroscopic fat can be detected on CT in renal cancers [1-3]. These include instances in which the renal sinus fat is engulfed by tumor and papillary cancers in which cholesterol necrosis has occurred [1-3].

\section{Diagnosis}

Renal carcinoma.

\section{Case 2: a 44-year-old female with hematuria (Fig. 2)}

\section{Description of findings}

A single image from an enhanced CT scan through the abdomen demonstrates multiple linear and wedgeshaped areas of decreased attenuation in both kidneys. These low attenuation areas do not exert any mass affect on adjacent spared renal parenchyma. In addition, the renal contours are irregular. Of note, there is no atherosclerotic calcification in the visualized segment of the abdominal aorta or the superior mesenteric artery.

\section{Discussion}

Linear and striated areas of low attenuation can be seen in patients with acute pyelonephritis and in patients with renal vascular injuries or infarcts. Renal parenchymal loss occurs as a long-term sequela of both processes, 


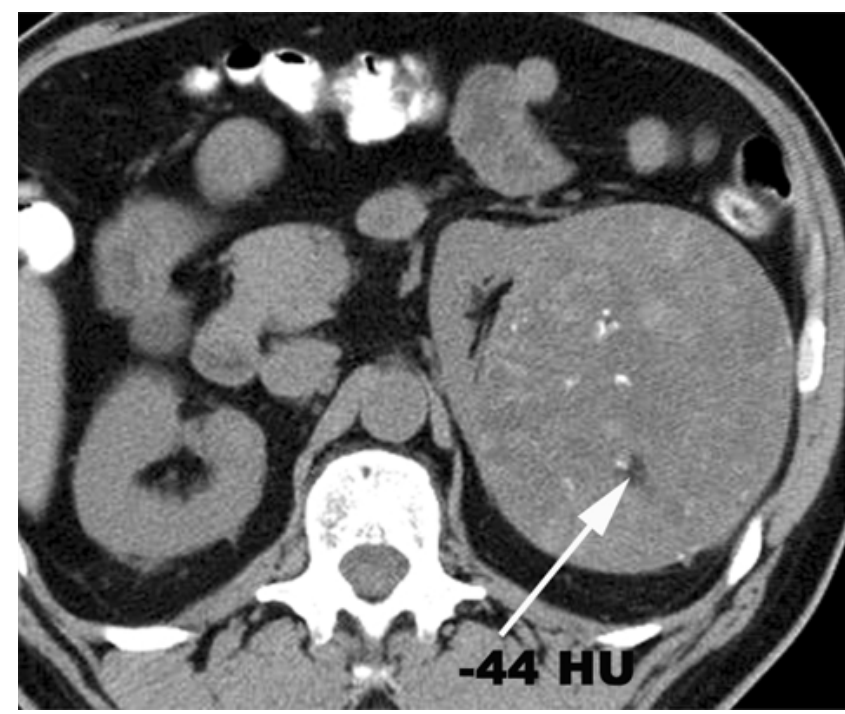

Fig. 1. A 47-year-old man with hematuria. Unenhanced CT image (case courtesy of Nirish Lal, MD, William Beaumont Hospital, Royal Oak, Michigan, USA).

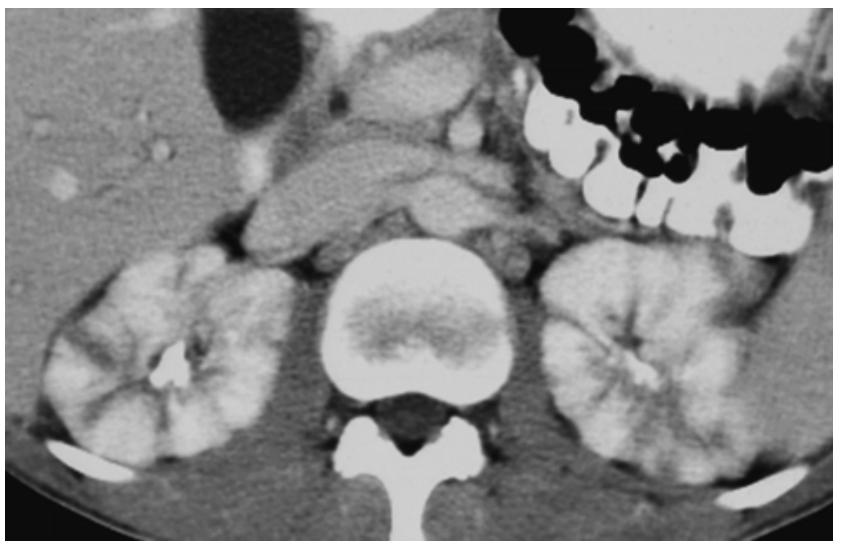

Fig. 2. A 44-year-old female with hematuria. Enhanced CT image.

although most cases of pyelonephritis resolve with minimal or no renal scarring if appropriate treatment is instituted. Usually, clinical distinction between pyelonephritis and embolic disease is possible. Although both entities can produce fevers and leukocytosis in the acute phase, most patients with acute pyelonephritis have pyuria, whereas those with renal infarcts do not. The provided history does not mention fever, high white blood cell count, or pyuria. The CT appearance and patient presentation in this instance are more likely due to multiple renal infarcts of different ages.

There are several possible etiologies of multiple renal infarctions, including atherosclerosis, septic emboli, and bland emboli such as may result from a cardiac dysrhythmia or a left ventricular aneurysm containing thrombus. In addition, multiple infarcts can occur as a result of vasculitis, one of the most common of which is polyarteritis nodosa. Other vasculitides that affect the kidneys include scleroderma and the vasculitis produced by methamphetamine drug use.

Polyarteritis nodosa is an autoimmune disease that typically results in medium and small vessel vasculitis. Patients with polyarteritis may present with fever, weight loss, and abdominal pain. The kidneys are affected in more than $80 \%$ of patients and are involved more often than the brain, liver, spleen, and lungs. On CT, multiple renal infarctions of different ages may be detected. The small aneurysms that form (and that may be seen with CT or conventional angiography) may also bleed and produce subcapsular and perinephric hematomas [4].

In this case, the absence of any other clinical or imaging findings suggests that a vasculitis is present, of which polyarteritis nodosa is the most common.

\section{Diagnosis}

Multiple renal infarctions from polyarteritis nodosa.

\section{Case 3: a 79-year-old female who is confused and acting toxic (Fig. 3)}

\section{Description of findings}

A plain abdominal radiograph (Fig. 3A) and an unenhanced CT image viewed with standard (Fig. 3B) and wide (Fig. 3C) windows demonstrate a collection of air in the left renal fossa that is intimately associated with the left kidney. There is no associated fluid.

\section{Discussion}

Air can occasionally be seen in the renal collecting systems, ureters, or bladder as a result of gas-forming infection of the urine, recent instrumentation, or fistula with adjacent bowel. In this case, however, gas is present within the substance of the kidney and not just the renal collecting system, thus establishing the diagnosis of emphysematous pyelonephritis. Further, there is no mention of recent surgery and it is stated that this patient is "acting toxic," a presentation strongly suggesting acute infection and supporting the imaging diagnosis.

Emphysematous pyelonephritis is a severe acute infection of the kidneys by a gas-producing organism, usually Escherichia coli (in 66\% of cases). Other identified pathogenic organisms include Klebsiella pneumoniae, Aerobacter aerogenes, Proteus mirabilis, and Candida tropicalis. The most commonly produced gases are carbon dioxide and hydrogen.

In some series, more than $90 \%$ of patients have a history of diabetes mellitus. Obstruction of the urinary tract is common but need not be present. Currently, the diagnosis is almost always made on the basis of imaging findings.

Two types of emphysematous pyelonephritis have been identified, one in which the air is located within 

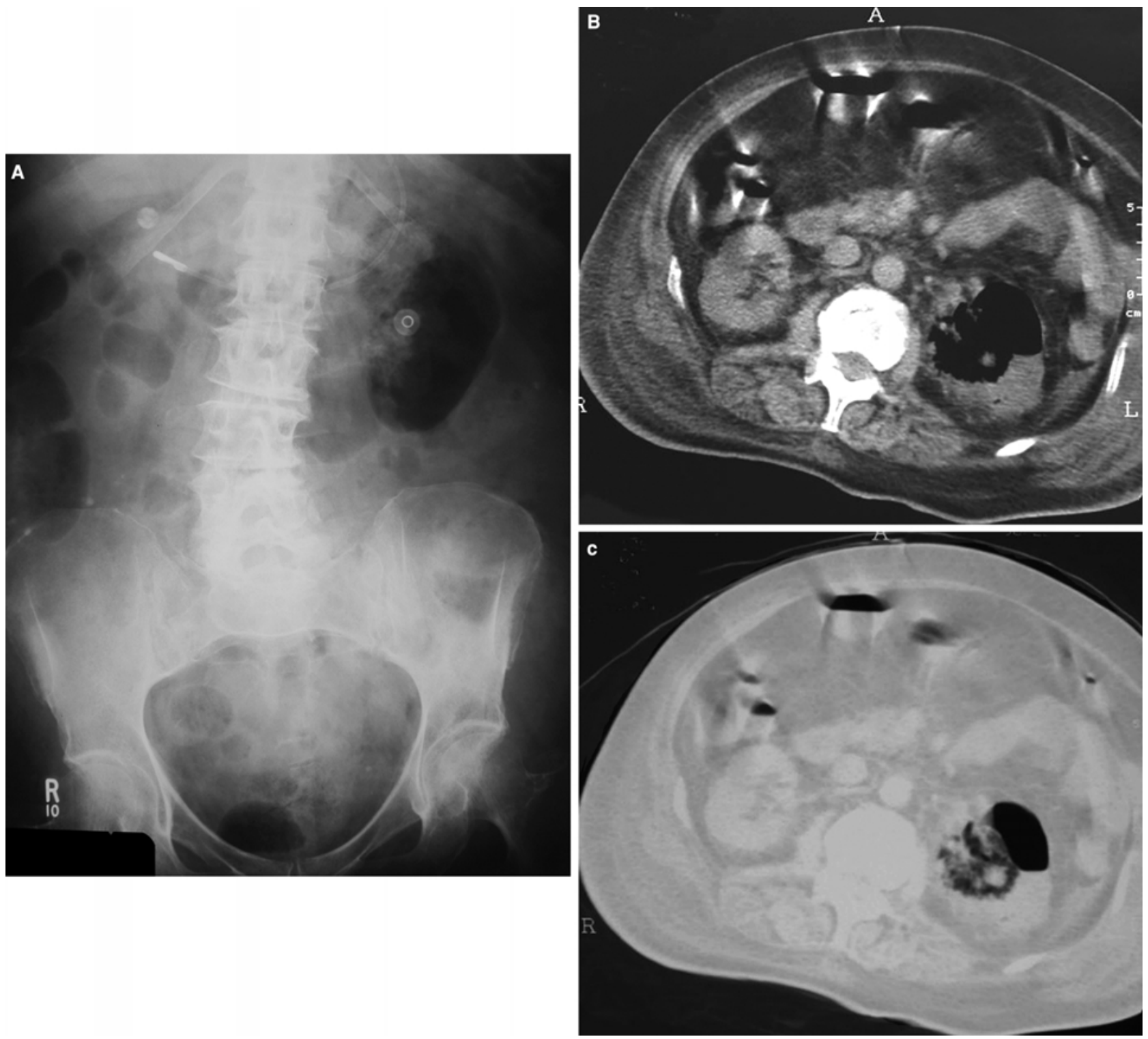

Fig. 3. A 79-year-old female who is confused and acting "toxic." A Plain abdominal radiograph. B, C Soft tissue and wide window presentations of the same unenhanced CT image.

loculated renal or perirenal fluid collections and the other in which mottled or streaky collections of air infiltrate into the renal parenchyma and in which there is little associated fluid [5]. The first type of emphysematous pyelonephritis, which has been shown to have a mortality rate of just below $20 \%$, can often be effectively treated with percutaneous drainage and large-dose intravenous antibiotics [5]. In contrast, morbidity and mortality rates from the second type are very high, approaching 70\% [5]. Treatment of these cases, therefore, is very aggressive, involving use of intravenous antibiotics and nephrectomy. Percutaneous drainage is not feasible because little or no drainable fluid is present.

\section{Diagnosis}

Emphysematous pyelonephritis.

\section{Case 4: a 25-year-old male, other history withheld (Fig. 4A)}

\section{Description of findings}

A single intravenous contrast-enhanced CT image through the midabdomen demonstrates normal perfusion of the left kidney, but nearly complete absence of perfusion of the imaged portion of the right kidney. Despite the lack of right renal perfusion, contrast material has been excreted into the right renal pelvis. 

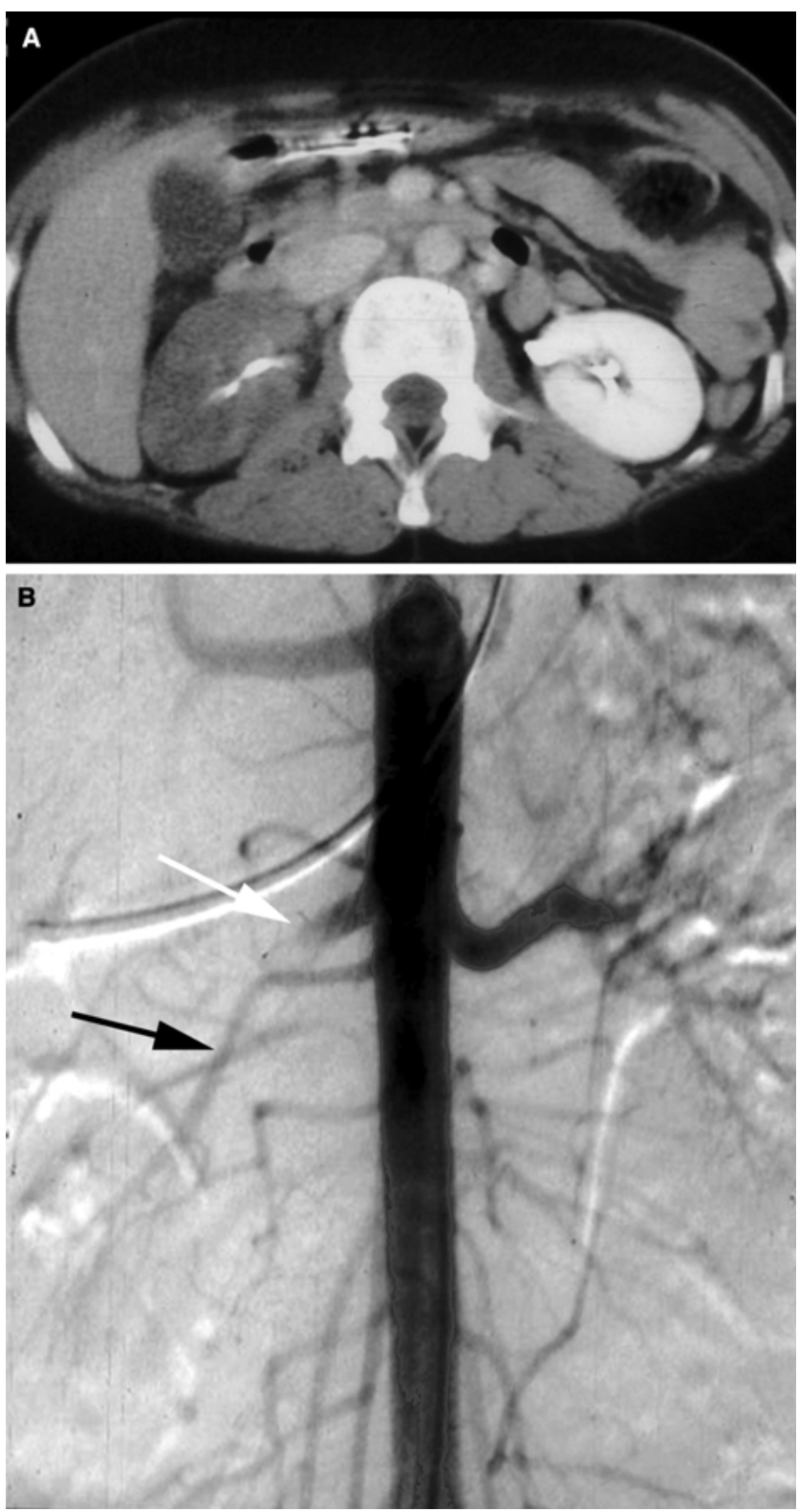

Fig. 4. A 25-year-old male. Other history withheld. A Enhanced CT image. B Image from a subsequently performed aortogram in the same patient demonstrates acute occlusion of the main right renal artery (white arrow). A smaller accessory right lower pole renal artery is still intact (black arrow).

\section{Discussion}

Complete absence of perfusion of a kidney indicates a major vascular insult to the kidney. Although severe renal artery stenosis can lead to renal artery occlusion, the affected kidney is usually quite small (from chronic hypoperfusion). In this case, the kidney is normal in size. Further, renal artery stenosis is usually due to atherosclerosis, which would be unusual in a young patient such as this, and no atherosclerotic vascular calcification is noted within the abdominal aorta on the provided CT image. Occasionally, an aortic dissection results in acute hypoperfusion of a kidney when its renal artery communicates with an underperfused false lumen. In this instance, no intimal flap is visualized in the imaged portion of the abdominal aorta. An acute embolus in the right renal artery (such as might occur in a patient with atrial fibrillation or a left ventricular aneurysm) is an additional, but very unusual, possibility. In a young patient, another consideration would be acute traumatic renal artery occlusion, usually due to blunt trauma.

Acute traumatic renal artery occlusion from blunt trauma results from a traumatic intimal tear in a renal artery (more commonly) or a complete renal pedicle avulsion [6]. These injuries are extremely rare but, when present, have a characteristic CT appearance, in which there is nearly complete nonperfusion of a kidney (as demonstrated by lack of intravenous contrast material enhancement of the renal parenchyma) [7]. CT is extremely sensitive in identifying these injuries. Diagnostic angiography is not required for confirmation of the CT findings. Renal pedicle injuries can be present with or without other visceral organ injuries and with or without the presence of any identifiable retroperitoneal hematoma. In the present case, no other injuries are present and there is no perinephric fluid.

Small components of the kidney may still be perfused after a renal pedicle injury [7]. Specifically, there can be residual enhancement of a rim of parenchyma due to preservation of flow in the renal capsular artery. The medulla may enhance minimally due to flow through periureteric and lumbar collateral arteries. Such collateral perfusion does not result in the prompt excretion of contrast material into the renal collecting system, as is demonstrated in this case. It is possible to see excretion into the renal collecting system, if only one of multiple renal arteries or only a renal artery branch is occluded. In the case presented here, this is exactly the situation. The larger of two right renal arteries has been traumatically occluded, as is demonstrated on an image from a subsequently performed arteriogram (Fig. 4B). A smaller lower pole right renal artery is still intact.

It is generally believed that, for renal salvage to be successful, surgical revascularization of a traumatically occluded renal artery must be performed within 6 to 12 $\mathrm{h}$ (warm ischemic time). Unfortunately, the renal salvage rate is extremely low. In a retrospective review of previously reported cases, only 34 of 139 patients with renal pedicle injuries underwent attempted revascularization. Of these 34 patients, surgery was successful in only nine instances (26\%) [8]. For this reason, urologists may elect to defer surgery in hemodynamically stable patients with properly functioning contralateral kidneys.

\section{Diagnosis}

Traumatic occlusion of one of two right renal arteries. 

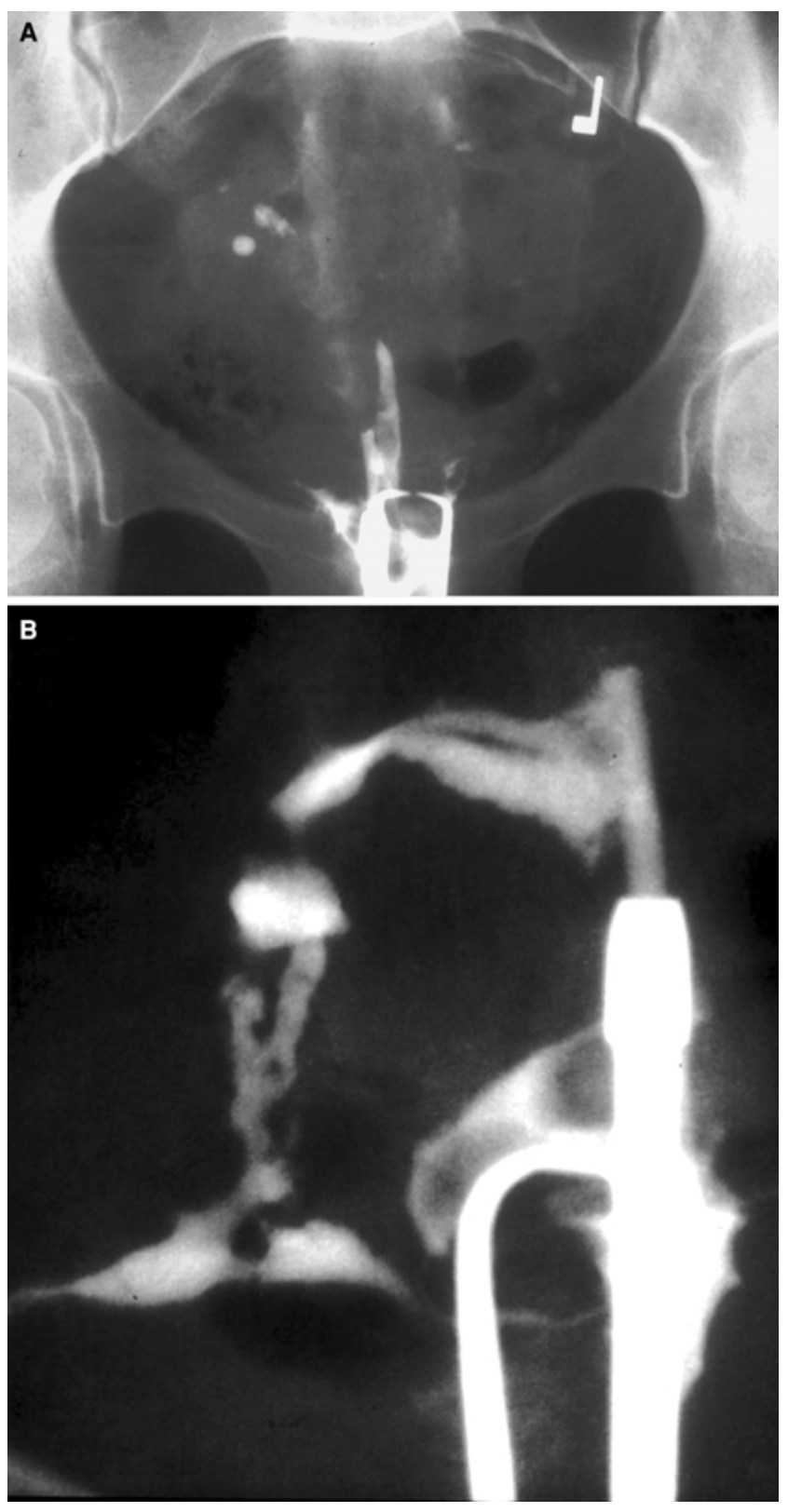

Fig. 5. A 37-year-old female with infertility. A Hysterosalpingogram. B Hysterosalpingogram in another patient demonstrates the multiple linear filling defects of uterine synechiae in the endometrial cavity. (The uterus is so severely anteverted that its fundus is inverted.)

\section{Case 5: a 37-year-old female with infertility (Fig. 5A)}

\section{Description of findings}

A single image from a hysterosalpingogram demonstrates a small amount of injected contrast material in the lower endocervical canal and additional contrast material leaking back into the vagina. The endometrial cavity is not filled. There is also no filling of the fallopian tubes and no free intraperitoneal spill.

\section{Discussion}

Occasionally, if the seal in the lower cavity or cervix is not complete during contrast material injection, contrast can flow back into the vagina and a normal endometrial cavity may not be opacified; however, if the hysterosalpingogram is properly performed, the endometrial cavity may not be opacified because it is completely scarred, with opposing walls of the endometrium adhering to one another, resulting in luminal obliteration. In this instance, there is no place for injected contrast material to go other than back out of the external os.

Endometrial adhesions or uterine synechiae most commonly occur after endometrial instrumentation (dilatation and curettage, abortion, or other uterine surgery, including caesarian section). Less commonly, they can form after pregnancy, trauma, or infection. On hysterosalpingography, these adhesions typically produce characteristic irregular, angulated, and sharply contoured filling defects in the endometrial cavity (Fig. 5B). Rarely, Gartner duct remnants in the cervical canal can produce linear filling defects that are similar in appearance to uterine synechiae; however, unlike uterine synechiae, such remnants are always present in the cervical canal and never present in the body or fundus of the uterus.

In some patients, synechiae are so severe that the endometrial cavity becomes completely obliterated, as is the case with this patient. This phenomenon has been called Asherman syndrome, although Asherman syndrome was originally defined merely as the clinical presentation of hypomenorrhea or amenorrhea after instrumentation [9].

Currently, the preferred treatment of uterine synechiae is hysteroscopic lysis of adhesions. This is a minimally invasive procedure and, if successful, theoretically can preserve patient fertility.

\section{Diagnosis}

Asherman syndrome.

\section{Case 6: a 44-year-old male from Columbus, Ohio, USA, with 10 months of progressive confusion, weakness, and lethargy (Fig. 6)}

\section{Description of findings}

A single intravenous enhanced CT image shows bilateral soft tissue attenuation adrenal masses. The spleen is enlarged but homogeneous. There are no masses in the visualized portions of the liver and no enlarged lymph nodes are identified.

\section{Discussion}

The differential diagnosis of bilateral adrenal masses is quite large and includes metastatic disease, bilateral li- 


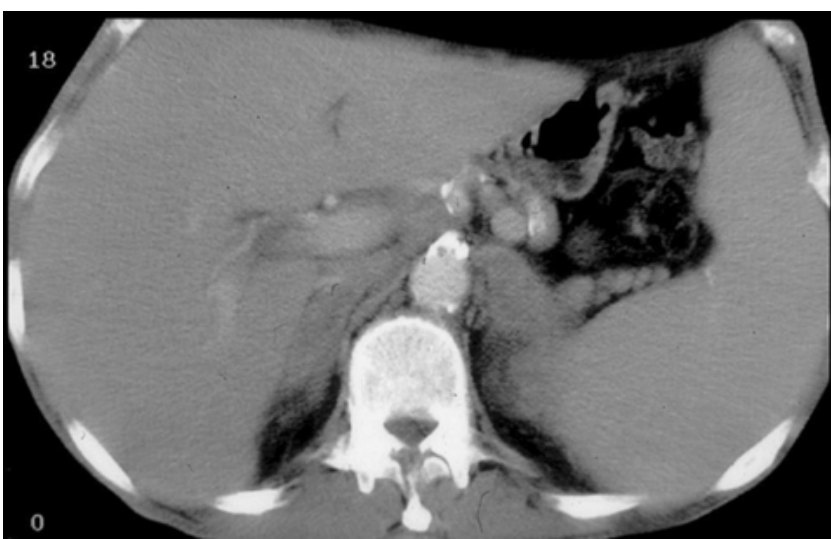

Fig. 6. A 44-year-old male from Columbus, Ohio, USA, with 10 months of progressive confusion, weakness, and lethargy. Enhanced CT image.

pid-poor adrenal adenomas, and, less commonly, adrenal lymphoma, adrenal hemorrhage, and granulomatous disease. In this patient, the provided history is extremely helpful. The described symptoms are characteristic of adrenal insufficiency. Adrenal insufficiency is only very rarely produced by the more common causes of adrenal masses, especially metastatic foci, and is not produced by adrenal adenomas.

Adrenal insufficiency can occur acutely, subacutely (with symptoms lasting for up to 2 years), or chronically (with symptoms lasting longer than 2 years). Acute adrenal insufficiency usually occurs as a result of adrenal hemorrhage (secondary to overwhelming sepsis/infection or trauma). In contrast, subacute and chronic adrenal insufficiency can result from autoimmune diseases, infection with human immunodeficiency virus, antiphospholipid syndrome (resulting in adrenal hemorrhage), and granulomatous disease (most often due to tuberculosis or histoplasmosis). Metastatic disease and adrenal lymphoma cause adrenal insufficiency only very rarely. Patients with acute and subacute adrenal insufficiency usually have bilateral adrenal enlargement, whereas patients with longstanding adrenal insufficiency tend to have small/atrophic adrenal glands that often contain calcification [10, 11].

It has been observed that "the combination of adrenal enlargement on CT and laboratory findings of adrenal insufficiency should suggest the diagnosis of histoplasmosis, especially in endemic areas" [11]. This patient lived in the Ohio Valley, a region where histoplasmosis is indeed endemic.

When the adrenal glands are acutely or subacutely involved with histoplasmosis, there is a tendency for the adrenal glands to maintain their normal shape but to become enlarged [11]. In comparison, metastatic disease usually produces rounded adrenal masses. Patients with granulomatous adrenal disease frequently have masses that demonstrate areas of diminished attenuation on CT, corresponding to areas of necrosis [11], which is suggested in this case by subtle central low attenuation.

\section{Diagnosis}

Adrenal insufficiency due to bilateral adrenal histoplasmosis.

\section{Case 7: follow-up of renal mass detected on a renal ultrasound (Fig. 7)}

\section{Description of findings}

Two magnetic resonance (MR) images of the right kidney are provided, one an in-phase and the other an outof-phase (opposed-phase) chemical shift image (the MR sequence of the latter image is revealed by the "India ink" artifact surrounding the kidney). There is a large right renal mass that is not clearly visible on the in-phase image, but that is quite conspicuous on the out-of-phase image, due to pronounced signal loss.

\section{Discussion}

The opposed-phase signal loss of the right renal mass indicates that there is a combination of lipid and fluid within the mass. This feature is not characteristic of angiomyolipomas, the most commonly imaged renal tumor containing macroscopic fat. Most angiomyolipomas do not contain fluid in the fat-containing regions and, therefore, do not lose signal on opposed-phase images. In addition, the macroscopic fat in angiomyolipomas is usually of very high signal on T1- and T2-weighted MR images. In this patient, the renal mass has a nearly identical signal to the normal renal parenchyma on the in-phase image.

Some renal cancers, particularly those of the clear cell type, contain abundant intracellular lipid, and it is the presence of this lipid in addition to water that causes signal loss on opposed-phase chemical shift images [12]. Thus, signal loss within a renal mass on opposed-phase images should be considered a worrisome sign and clear cell renal carcinoma has to be considered.

\section{Diagnosis}

Clear cell renal carcinoma.

\section{Case 8: a 63-year-old female with right loin pain and microscopic hematuria (Fig. 8)}

\section{Description of findings}

Two images from a CT urogram are provided. The first is a coronal reformatted image of the renal collecting systems and ureters, and the second is an axial image through the midportions of the right upper pole ureter 

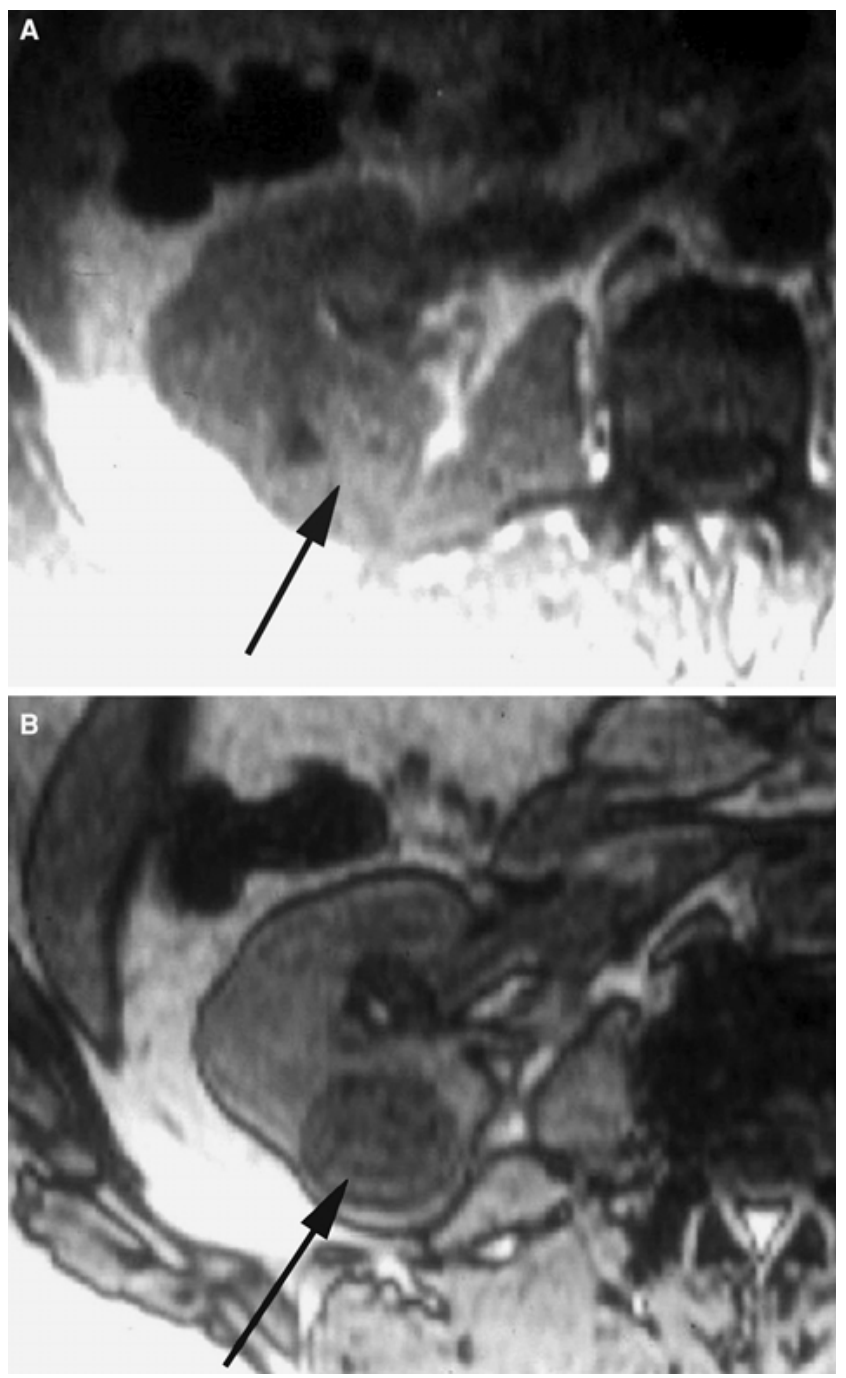

Fig. 7. Follow-up of renal mass detected on ultrasonography. A, B MR images from two different sequences (case courtesy of Cary Siegel, MD, Mallinckrodt Institute of Radiology, St. Louis, Missouri, USA).

and the single left ureter. Despite the presence of high attenuation excreted contrast material in the right renal collecting systems, a rounded high attenuation focus is noted in a large extrarenal pelvis that drains the lower pole moiety of a duplicated collecting system. The visualized portions of the right ureter from the upper pole moiety appear normal, aside from the presence of a small kink. There is no evidence of a ureteral filling defect of mass. In comparison, a tiny soft tissue lesion can be seen in the midureter on the axial image.

\section{Discussion}

Multidetector CT urography (MDCTU) is currently thought to be the most sensitive comprehensive imaging examination for evaluation of the entire urinary tract [13]. Preliminary work has suggested that MDCTU can
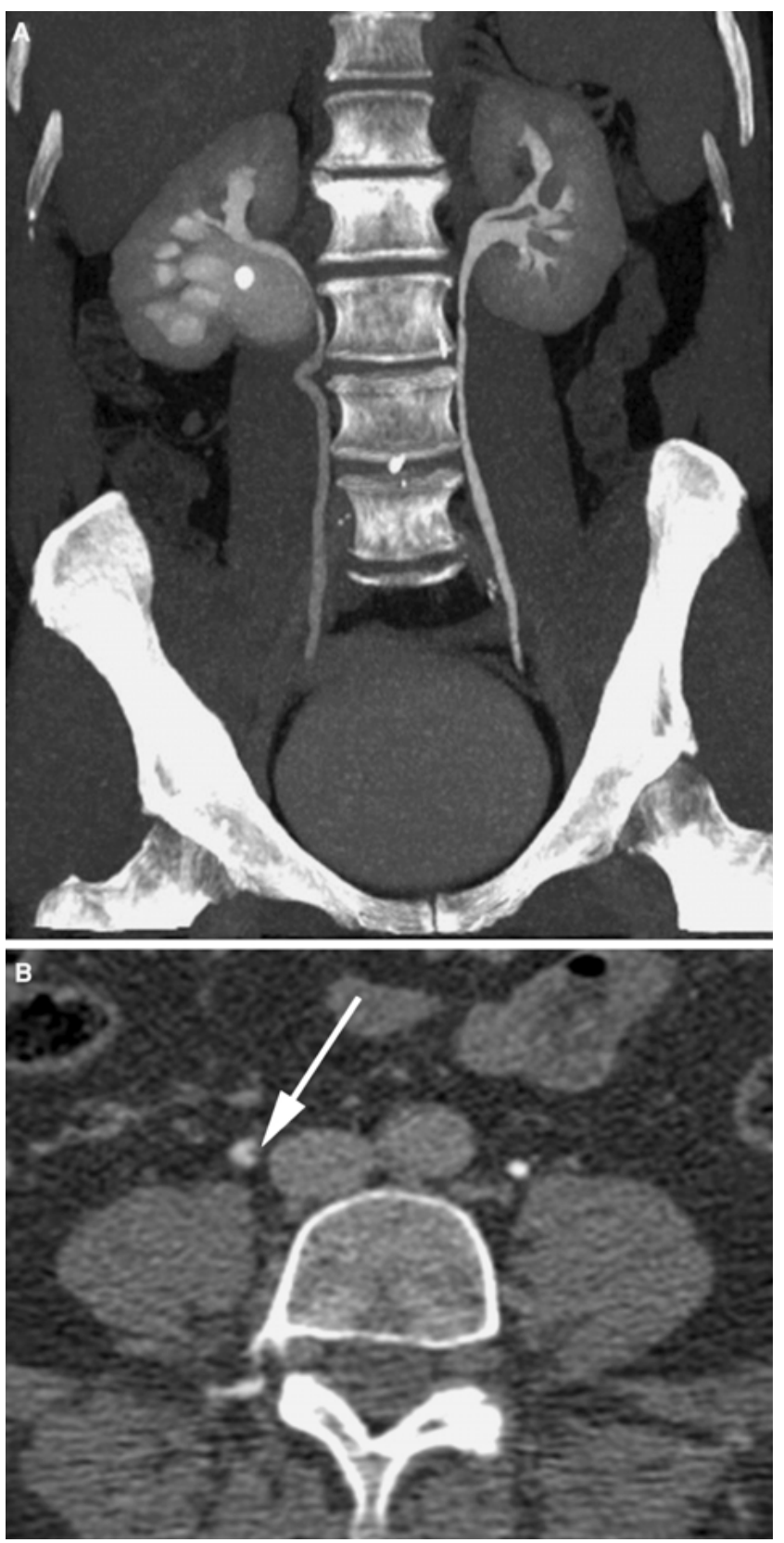

Fig. 8. A 63-year-old female with right loin pain and hematuria. A Coronal reformatted and $\mathbf{B}$ axial images from the excretory phase series of a CT urogram.

detect many different urinary tract abnormalities once thought to be invisible on CT, including tiny uroepithelial neoplasms [13]. In one series, urinary tract abnormalities were detected on MDCTU with greater sensitivity than on retrograde pyelography [14], a finding that suggests that MDCTU should also be superior to excretory urography. The present case demonstrates a tiny $(1$ to $2 \mathrm{~mm}$ ) soft tissue attenuation filling defect in the mid right ureter that drains the upper pole moiety. A uroepithelial neoplasm is the most likely diagnosis. 


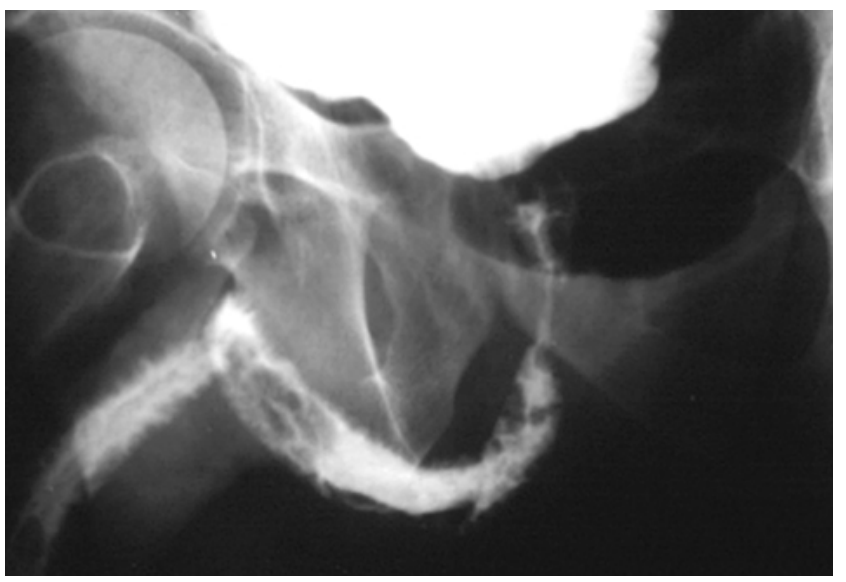

Fig. 9. A 35-year-old male with 2 years of painless bloody spotting from urethra after intercourse. Urethrogram.

As previously mentioned, this study also demonstrates a duplicated right renal collecting system with a calculus in the lower pole moiety renal pelvis. Although urinary tract calculi sometimes be identified on the excretory phase images (as in this case), on many occasions they are obscured by the densely excreted contrast material. In nearly all cases, however, calculi are easily detected on the unenhanced images, which are obtained as the first of the two or three utilized series included in most MDCTU examinations.

\section{Diagnosis}

Large right renal pelvic calculus in the lower pole moiety of a duplicated right renal collecting system and ureteral uroepithelial neoplasm in the ureter of the right upper pole moiety.

\section{Case 9: a 35-year-old male with 2 years of painless bloody spotting from the urethra after intercourse and, occasionally, after urination; no history of trauma (Fig. 9)}

\section{Description of findings}

A single oblique image from an urethrogram demonstrates marked irregularity in the contour of nearly the entire urethra but which is most pronounced in the anterior urethra. There are large, rounded, and linear filling defects within the urethral lumen. There is no evidence of a urethral stricture.

\section{Discussion}

Most nontraumatic urethral pathology relates to stricture disease resulting from inflammation, with most infections occurring secondary to gonorrhea, tuberculosis, or nonspecific urethritis. However, no stricture has formed in this patient, although, given the long segment of involved urethra, inflammation remains very likely. Another inflammatory process, one that produces multiple intraluminal masses, must be considered $[15,16]$.

Condyloma acuminata of the male urethra affects up to $5 \%$ of men with condyloma, all of whom have cutaneous manifestations [15-17]. The presence of this disease is always clinically obvious. The direct spread of venereal warts into the urethral lumen has a distinctive urethrographic appearance (as is seen in this case), although the differential diagnosis should still include urethral carcinoma (which only rarely affects such a long segment of the urethra), metastatic disease (e.g., from melanoma, which is also exceedingly rare), and other types of urethritis (including urethritis cystica).

When retrograde urethrography is performed in these patients, there is some risk of proximal urethral or bladder seeding. Such seeding is even more likely when endoscopy is performed. For this reason, some recommend that, if imaging evaluation is necessary, cystourethrography after intravenous administration of contrast material is preferred [15].

Current treatment of urethral warts includes excision and fulguration, combined with urethral infusions of podophyllin, thiotepa, or 5-fluorouracil. Several treatment sessions may be needed [17].

\section{Diagnosis}

Condyloma acuminata of the entire male urethra.

\section{Case 10: an 18-year-old male with gross hematuria and intermittent flank pain (Fig. 10)}

\section{Description of findings}

Two images from an excretory urogram are provided, the first an anteroposterior view of the left renal collecting system and ureter and the second a coned-down left posterior oblique view of a very abnormal left ureter. Both images demonstrate a large irregularly contoured filling defect in the mid left ureter. The ureter surrounding and more proximal to the filling defect is dilated. There is also left pelvocaliectasis and proximal ureterectasis. In addition, there is an unusual deformity whereby the proximal portion of the left ureter appears to invaginate into a more dilated midportion of the left ureter (the focus of Fig. 10B).

\section{Discussion}

The unusual deformity of the proximal and midureter has an appearance diagnostic of an intussusception. This intussusception appears to be caused by a large ureteral filling defect, which serves as a lead point. Differential 

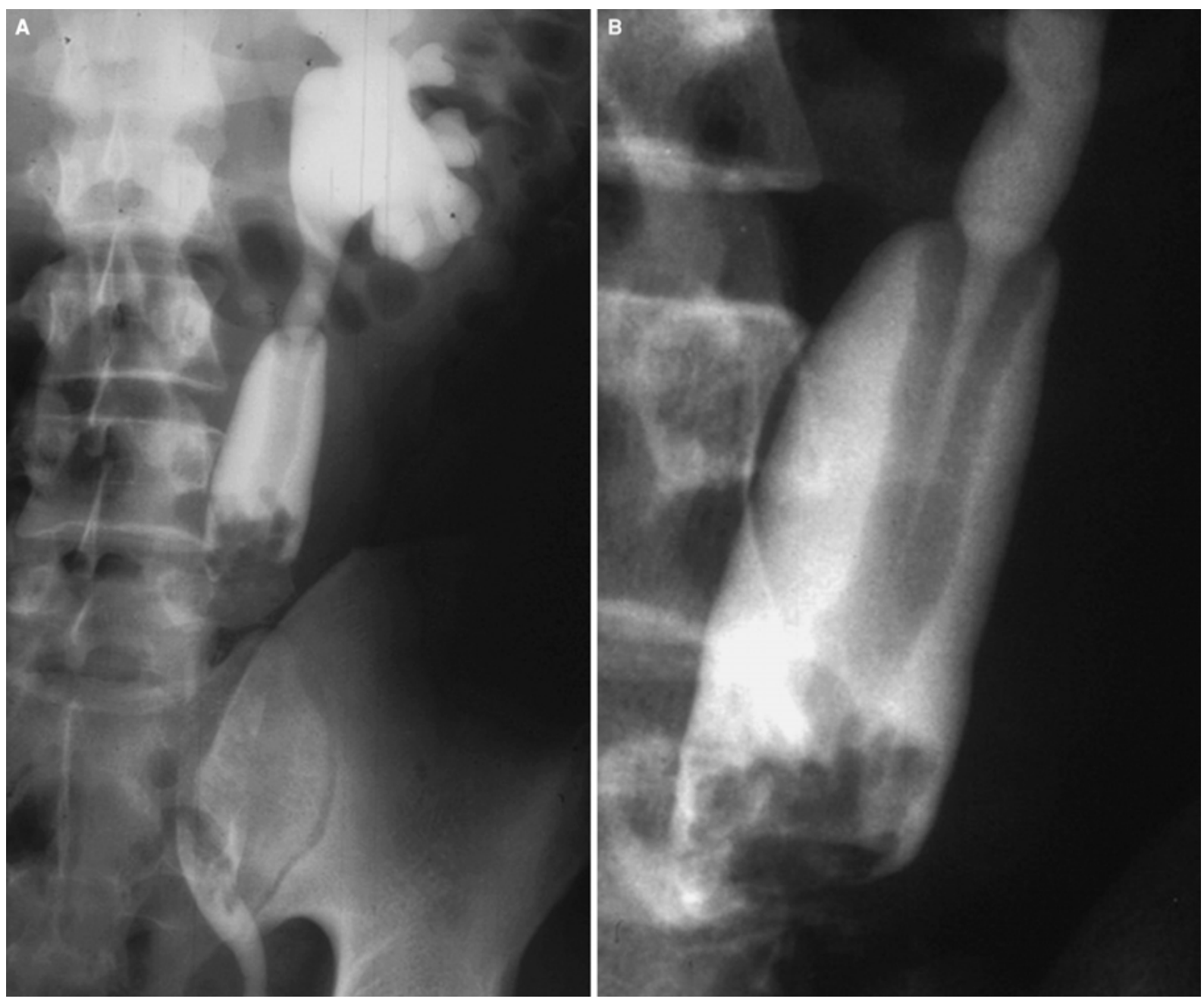

Fig. 10. An 18-year-old male with gross hematuria and intermittent flank pain. A, B Two different images at different magnifications from an excretory urogram.

diagnosis for the lead point includes transitional cell carcinoma or other more unusual uroepithelial tumor, including a fibroepithelial polyp.

As with bowel intussusceptions, ureteral intussusceptions occur when a mass, serving as a lead point, is propelled from the proximal portion of a ureter into a more distal portion by normal peristalsis. Intussusception of ureteral tumors is very rare, with only a few published reports [18, 19]; however, the appearance is so characteristic that when present the diagnosis can usually be made with confidence. Although a variety of ureteral neoplasms may intussuscept, all reported intussusceptions have involved low-grade, slow-growing tumors. This is because high-grade infiltrative tumors are adherent to the ureteral wall and cannot serve as lead points. For this reason, although transitional cell carcinomas of the ureter are much more common than fibroepithelial polyps, intussusceptions more commonly involve fibroepithelial polyps [18]. In addition, transi- tional cell carcinoma would be unlikely in this case because these neoplasms are uncommon in young adults. Although fibroepithelial polyps are occasionally encountered in middle-age adult patients, many patients are younger, often younger than 20 years [20-22].

Fibroepithelial polyps of the ureter are rare, usually solitary, benign neoplasms [21-23]. Although their etiology is not well defined, some have speculated that ongoing irritation and inflammation are often responsible for their development [20]. Pathologic analysis demonstrates that these tumors consist of a connective tissue stalk covered by normal transitional epithelium [23]. They range in length from $3 \mathrm{~mm}$ to $13 \mathrm{~cm}$, but usually measure between 2 and $5 \mathrm{~cm}$ [20]. Fibroepithelial polyps are at risk for producing an intussusception, in part because they often have a long stalk that connects them to their point of origin.

Treatment of an intussuscepting fibroepithelial polyp requires open segmental resection. In contrast to treat- 


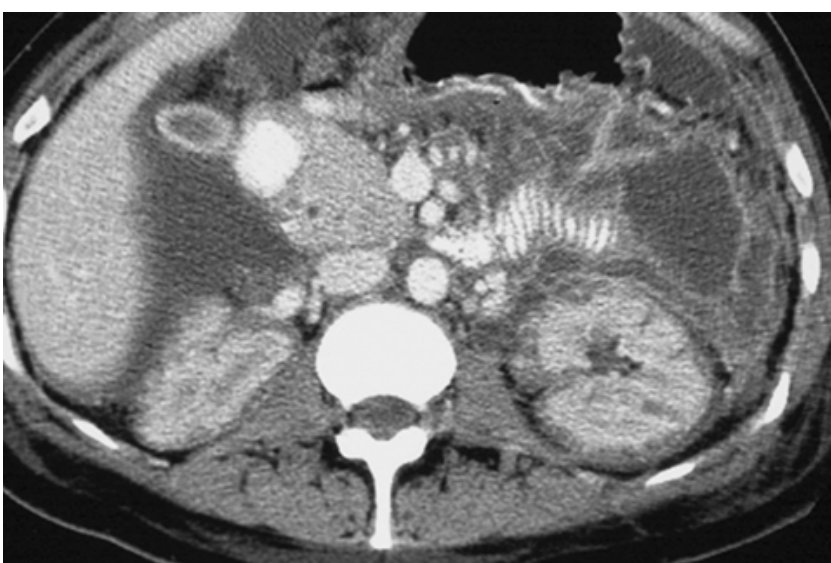

Fig. 11. Elderly female with severe pancreatitis. CT with oral and intravenous contrast material performed to evaluate for abscess.

ment of nonintussuscepting polyps, ureteroscopic resection is not possible.

\section{Diagnosis}

Ureteroureteral intussusception with a fibroepithelial polyp as a lead point.

\section{Case 11: elderly female with severe pancreatitis; CT performed to evaluate for abscess (Fig. 11)}

\section{Description of findings}

A single oral and intravenous contrast-enhanced CT image through the midabdomen reveals a nearly uniform rim of low attenuation surrounding the periphery of the visualized portions of both kidneys. This rim of low attenuation is surrounding heterogeneously enhancing renal medulla and in turn is surrounded (albeit incompletely) by a thin peripheral rim of increased attenuation. There are multiple large loculations of peripancreatic fluid in the left anterior pararenal space. Free intraperitoneal fluid is present adjacent to the gallbladder and medial to the liver.

\section{Discussion}

The CT findings in both kidneys are diagnostic of acute cortical necrosis. Characteristic CT features include (a) nonenhancement of the renal cortex, (b) enhancement of the renal medulla, and (c) absent excretion into the renal collecting system [24, 25]. Enhancement of a thin rim of capsular tissue may also be seen peripheral to the infarcted renal cortex (due to preservation of flow in the renal capsular artery), as in this case [26].

Acute cortical necrosis is a rare cause of acute renal failure, usually occurring in extremely ill individuals, often as a result of obstetric complications, hemorrhagic shock, disseminated intravascular coagulation, severe trauma, sepsis, shock, or burns. As the term implies, affected patients develop necrosis of the renal cortex with sparing of the medulla. Necrosis develops as a result of constriction of small intracortical blood vessels [26] with preferential flow of blood away from the renal cortex, a phenomenon that can be seen in severely ill patients with hypotension. The likelihood that normal renal function will return is extremely low. Usually, over time, the involved kidney becomes shrunken and scarred. Cortical nephrocalcinosis may then develop.

\section{Diagnosis}

Acute cortical necrosis secondary to severe acute pancreatitis.

\section{Case 12: a 54-year-old male with urinary tract infection (Fig. 12)}

\section{Description of findings}

A single coned-down image from an excretory urogram reveals a normal-appearing right kidney and normal right renal calices, infundibula, and right renal pelvis. The ureter is normal in course and caliber and no filling defects are identified within it; however, there is a small diverticulum protruding cephalically from the lateral aspect of ureter.

\section{Discussion}

There are two different causes of diverticular-like projections from the ureter: ureteral pseudodiverticulosis and ureteral bud.

Ureteral pseudodiverticulosis represents a rare inflammatory reaction of the ureter in which relatively short outpouchings are noted to project from the ureteral lumen. Ureteral pseudodiverticulosis usually results in the formation of multiple diverticula and is most often bilateral [27]. An association between ureteral pseudodiverticulosis and uroepithelial neoplasms has been reported [27].

A ureteral bud, an even more rarely encountered abnormality, consists of a blind-ending partial duplication of the ureter, which can arise from the bladder or any portion of the ureter [28]. Ureteral buds represent true diverticula because all three layers of the ureteral wall are preserved. In contrast to ureteral pseudodiverticula, ureteral buds are nearly always solitary and are always more than twice as long as they are wide [28, 29]. Although ureteral pseudodiverticula generally appear to arise from the ureter at right angles to the long axis of the ureter, ureteral buds generally join the ureter at an acute angle, with their blind ends nearly always located cephalic to their origin. 


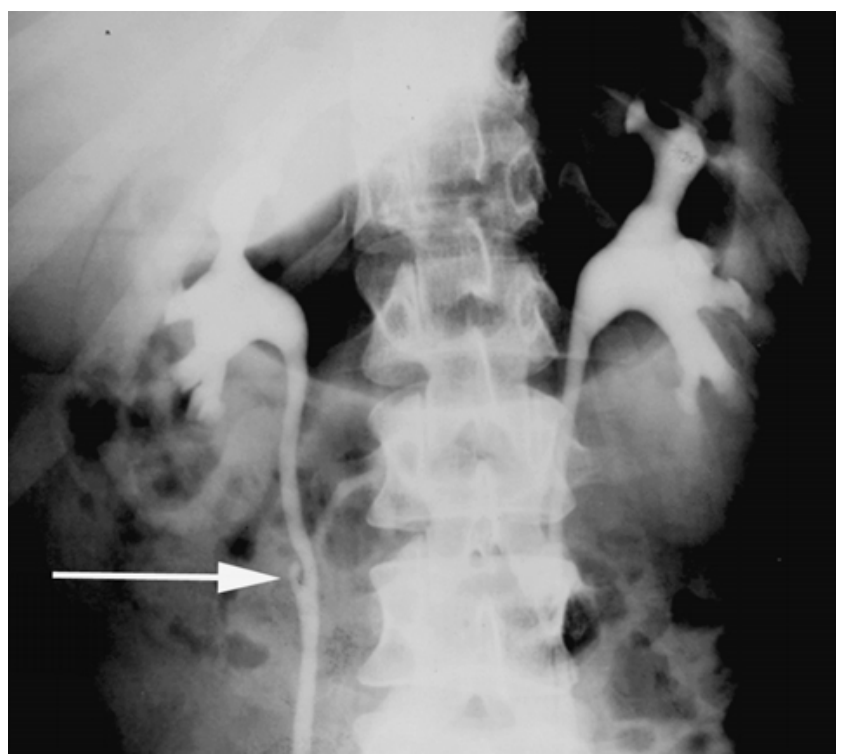

Fig. 12. A 54-year-old male with urinary tract infection. Image from an excretory urogram.

Ureteral buds do not usually cause any symptoms and may be found incidentally on excretory or CT urography [30]. On occasion, they may serve as a nidus for ongoing infection [29].

The ureteral projection in the present case is much more characteristic of a ureteral bud than of a pseudodiverticulum (because of the length of the projection, its orientation, and its angulation with the ureter). The patient history of urinary tract infection is not particularly helpful because infections can be seen in patients with both entities.

\section{Diagnosis}

Blind-ending ureteral bud (blind-ending ureteral duplication).

\section{Case 13: a 58-year-old male with chronic low-grade fevers (Fig. 13)}

\section{Description of findings}

Two intravenous contrast-enhanced CT images through the midabdomen demonstrate marked abnormality of the entire visualized left kidney, which is asymmetrically enlarged. The renal parenchyma contains several large areas of low attenuation rimmed by many punctate calcifications. There is extensive inflammatory stranding in the perinephric and peripelvic fat. Although scans were obtained in the excretory phase (as evidenced by the presence of contrast material in the right renal collecting system), there is no left renal excretion. Small calcified masses are identified in both psoas muscles. The lumbar vertebral body has an unusual orientation, particularly as seen on the first image.
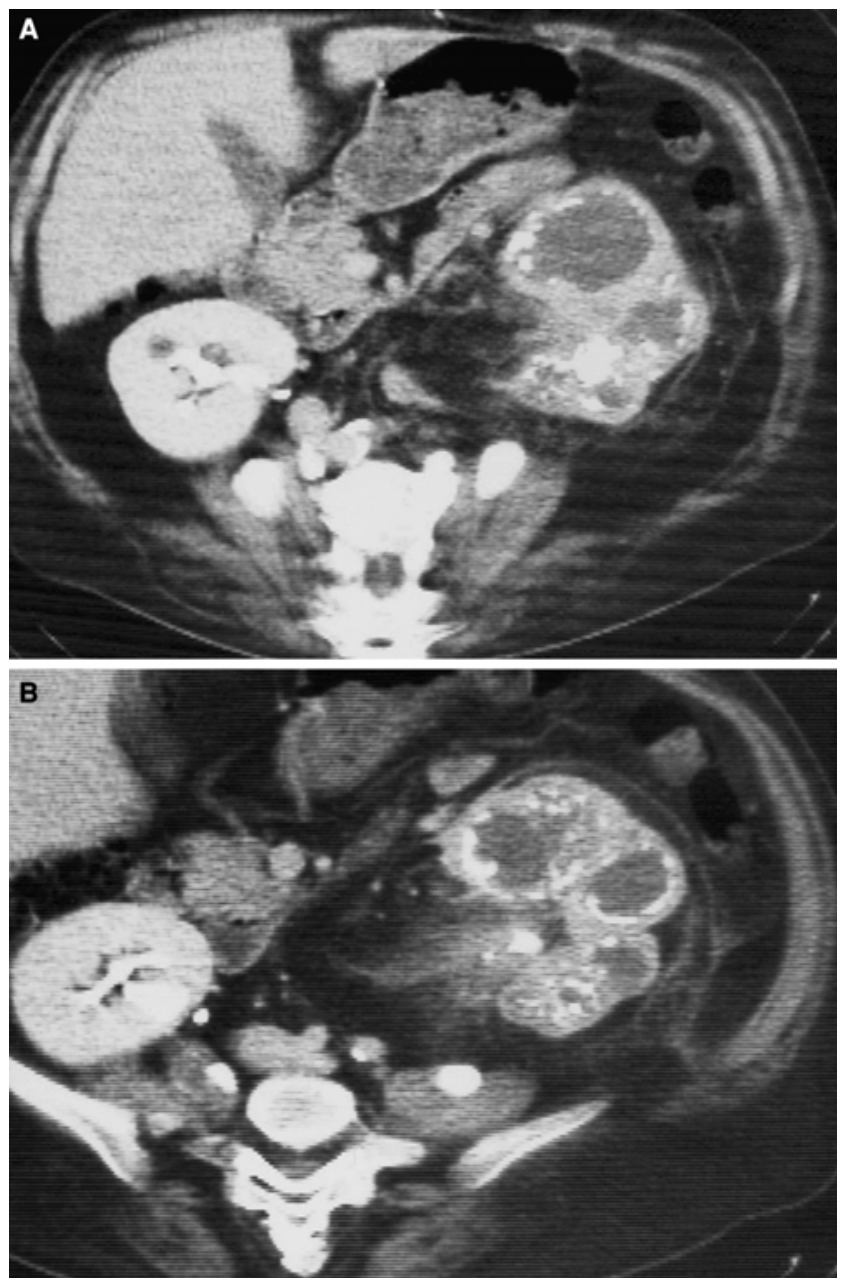

Fig. 13. A 58-year-old male with chronic low-grade fevers. A, B Enhanced CT images.

\section{Discussion}

The CT appearance suggests severe diffuse inflammation rather than a renal neoplasm. Xanthogranulomatous pyelonephritis (XGP), a chronic inflammatory process characterized by the presence of lipid-laden macrophages, most commonly affects the entire kidney, as seen in this instance. Also, as in this instance, in diffuse XGP there are large low attenuation collections in the kidney, the affected kidney is enlarged, there is no contrast excretion in the affected kidney, and there is often inflammatory spread into the perinephric regions [31]. However, the calcification in XGP, if present, usually is due to a large centrally located calculus, often staghorn in type [31]. Instead, in this patient, multiple tiny peripheral calcifications are present.

In this patient, the punctate calcifications, the calcified psoas masses, and the spinal deformity are much more suggestive of renal tuberculosis. Renal tuberculosis usually results from hematogenous seeding of the kidneys from disease in the lungs; the seeding occurs at the 


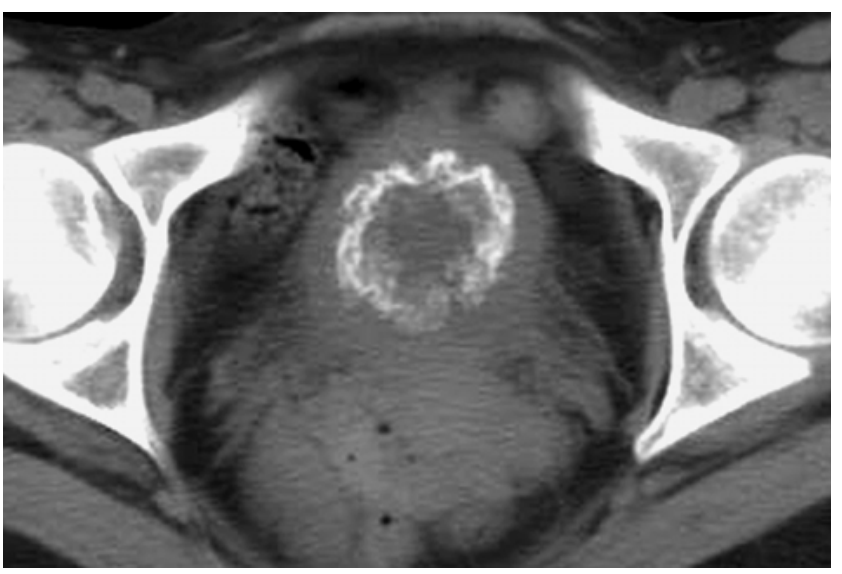

Fig. 14. A 37-year-old with lymphoma. Unenhanced CT image.

time of the initial lung infection. The renal disease remains quiescent until there is an insult to the host's immunity, at which time reactivation occurs.

The radiographic appearance of renal tuberculosis is very variable, although calcifications and large low attenuation areas (abscesses resulting from caseating necrosis) are frequently present $[32,33]$. The end stage of renal tuberculosis is autonephrectomy, whereby the entire kidney may become small, scarred, and densely calcified [32].

Tuberculosis can also involve the lumbar vertebral bodies and other soft tissues, with formation of calcifications in the areas of chronic abscess formation, the socalled cold abscesses.

\section{Diagnosis}

Renal and lumbar vertebral body tuberculosis with tuberculous "cold" abscesses in both psoas muscles.

\section{Case 14: a 37-year-old British male with lymphoma (Fig. 14)}

\section{Description of findings}

A single unenhanced CT image through the pelvis demonstrates coarse calcification in the wall of a relatively small-capacity bladder.

\section{Discussion}

There are several causes of diffuse bladder wall calcification. Globally, the most common cause of bladder calcification is Schistosomiasis, which results from migration of Schistosomiasis haematobium from the heart (after penetration of the skin and circulation through the venous system) to the venous plexus surrounding the bladder [34-36]. Bladder wall calcification is observed in many patients infected with S. haemato- bium. Calcification can be thin or thick, circumferential or focal $[35,36]$. Because the calcification is submucosal, it may develop in a bladder that retains its normal size and capacity, at least initially. Over time, however, the bladder capacity decreases as reactive fibrosis increases. Schistosomiasis is widely prevalent in some parts of the world, particularly in Africa, but is not found in the continental United States or Western Europe [34-36]. For this reason alone. Schistosomiasis would be unlikely in the present patient.

Although calcifications can occur in some uroepithelial neoplasms, these are usually focal [34-36]. Other causes of diffuse bladder wall calcification include tuberculosis (an occasional cause of cystitis, but one that rarely produces bladder wall calcification), alkaline-encrusted cystitis (a chronic cystitis usually encountered in an immunocompromised patient with a preexisting bladder abnormality who has undergone prior instrumentation), the sequelae of radiation therapy (radiation cystitis), and the sequela of chemotherapy (hemorrhagic cystitis) [35-39]. The last entity is very likely in this patient because cyclophosphamide, the agent that most commonly causes hemorrhagic cystitis, is routinely used to treat lymphoma.

Hemorrhagic cystitis occurs in up to $40 \%$ of patients treated with cyclophosphamide. Symptoms usually begin during or just after completion of therapy. Usually, the symptoms resolve without long-term sequelae; however, chronic changes in the bladder, including calcification, can occur in some instances [36, 38].

\section{Diagnosis}

Bladder wall calcification secondary to cyclophosphamide-induced hemorrhagic cystitis.

\section{References}

1. Helenon O, Chretien Y, Paraf F, et al. (1993) Renal cell carcinoma containing fat: demonstration with CT. Radiology 188:429-430

2. Strotzer M, Lehner KB, Becker K (1993) Detection of fat in a renal cell carcinoma mimicking angiomyolipoma. Radiology 188:427428

3. Radin DR, Chandrasoma P (1992) CT demonstration of fat density in renal cell carcinoma. Acta Radiol 33:365-367

4. Hekali P, Kivisaari L, Standerskjold-Nordenstam CG, et al. (1985) Renal complications of polyarteritis nodosa: CT findings. J Comput Assist Tomogr 9:333-338

5. Wan YL, Lee TY, Bullard MJ, Tsai CC (1996) Acute gas-producing bacterial renal infection: correlation between imaging findings and clinical outcome. Radiology 198:433-438

6. Cass AS, Luxenberg M (1987) Management of renal artery injuries from external trauma. J Urol 138:266-268

7. Sclafani SJ, Goldstein AS, Panetta T, et al. (1985) CT diagnosis of renal pedicle injury. Urol Radiol 7:63-68

8. Haas CA, Spirnak JP (1998) Traumatic renal artery occlusion: a review of the literature. Tech Urol 4:1-11

9. Winfield AC, Wentz AC (1992). Diagnostic imaging and, infertility 2nd ed. Baltimore: Williams and Wilkins, 151-166

10. Pozzi Mucelli RS, Pozzi Mucelli F, Muner G (1985) Computed tomographic follow-up in a case of Addison's disease. Eur J Radiol $5: 221-223$ 
11. Levine E (1991) CT evaluation of active adrenal histoplasmosis. Urol Radiol 13:103-106

12. Outwater EK, Bhatia M, Siegelman ES, et al. (1997) Lipid in renal clear cell carcinoma: detection on opposed-phase gradient-echo MR images. Radiology 205:103-107

13. Caoili EM, Cohan RH, Korobkin M, et al. (2002) Urinary tract abnormalities: initial experience with multi-detector row CT urography. Radiology 222:353-360

14. McCarthy CL, Cowan NC (2002) Multidetector CT urography (MD-CTU) for urothelial imaging. Radiology 225(P):237

15. Pollack HM, DeBenedictis TJ, Marmar JL, Praiss DE (1978) Urethrographic manifestations of venereal warts (condyloma acuminata). Radiology 126:643-646

16. Sawczuk I, Badillo F, Olsson CA (1985) Condylomata acuminata: diagnosis and follow-up by retrograde urethrography. Urol Radiol 5:273-274

17. Wasserman N (2000) Urethral neoplasms. In: Pollack HM, McClennan BL (eds). Clinical urography. 2nd ed. Philadelphia: WB Saunders, pp 1701-1702

18. Moretti KL, Jose JS (1987) Ureteral intussusception owing to a malignant ureteral polyp. J Urol 173:493-494

19. Fukushi Y, Orikasa S, Takeuchi M (1983) A case of ureteral intussusception associated with ureteral polyp. J Urol 129:1043-1044

20. Ugras S, Odabas O, Aydin S, et al. (1997) Fibroepithelial polyp of the ureter associated with an adjacent ureteral calculus. Int Urol Nephrol 29:543-549

21. DeBruyne FMJ, Moonen WA, Daenekindt AA, et al. (1980) Fibroepithelial polyp of the ureter. Urology 16:355-359

22. Liddell RM, Weinberger E, Schofield DE, et al. (1991) Fibroepithelial polyp of the ureter in a child. AJR 157:1273-1274

23. Bellin MF, Springer O, Mourey-Gerosa I, et al. (2002) CT diagnosis of ureteral fibroepithelial polyp. Eur Radiol 12:125-128

24. Goergen TG, Lindstorm RR, Tan H, et al. (1981) CT appearance of acute cortical necrosis. AJR 131:176-177

25. Jordan J, Low R, Jeffrey RB (1990) CT findings in acute renal cortical necrosis. J Comput Assist Tomogr 14:155-156

26. Lantsberg S, Rachinsky I, Lupu L, et al. (2000) Unilateral acute renal cortical necrosis: correlative imaging. Clin Nucl Med 25:184-186
27. Wasserman NF, La Pointe S, Posalaky IP (1985) Ureteral pseudodiverticulosis. Radiology 155:561-566

28. Culp OS (1947) Ureteral diverticulum: classification of the literature and report of an authentic case. J Urol 58:309-326

29. Nino-Murcia M, Friedland GW, deVries PA (2000) Congenital anomalies of the papillae, calyces, renal pelvis, ureter, and ureteral orifice. In: Pollack HM, McClennan BL, (eds). Clinical urography. 2nd ed. Philadelphia: WB Saunders, pp 791-793

30. Finder CA, Love L, Rich JI (1984) Blind ending ureters - clinical significance? Urol Radiol 4:235-238

31. Claes H, Vereecken R, Oyen R, et al. (1987) Xanthogranulomatous pyelonephritis with emphasis on computerized tomography scan. Urology 9:389-393

32. Goldman SM, Fishman EK, Hartman DS, et al. (1985) Computed tomography of renal tuberculosis and its pathological correlates. J Comput Assist Tomogr 9:771-776

33. Izbudak-Oznur I, Sozen S, Isik S (2002) Renal tuberculosis mimicking xanthogranulomatous pyelonephritis: ultrasonography, computed tomography and magnetic resonance findings. Turk $\mathrm{J}$ Pediatr 44:168-171

34. Fataar S, Rudwan M, Bassiony H, Satyanath S (1990) CT of genitourinary calcification due to schistosomiasis. Australas Radiol 34:234-237

35. Webb JAW (2000) Lower urinary tract calculi, calcifications. In: Pollack HM, McClennan BL (eds). Clinical urography. 2nd ed. Philadelphia: WB Saunders, pp 2252-2254

36. Dunnick NR, Cochran ST, Cohan RH, et al. Radiation cystitis. In: Genitourinary tract disease (fifth series) test and syllabus (\#43). Reston, VA: American College of Radiology, 1998:122145

37. Pollack HM, Banner MP, Martinez LO, Hodson CJ (1981) Diagnostic considerations in urinary bladder wall calcification. AJR 136:791-797

38. Francis RS, Schackelford GD (1974) Cyclophosphamide cystitis with bladder wall calcification. J Can Assoc Radiol 25:324-326

39. Verguts L, Deconinck K, Mortelmans LL (1987) Alkaline encrusting cystitis. Urol Radiol 9:53-55 